\title{
Generalized Helices on a Lightlike Cone in 3-Dimensional Lorentz-Minkowski Space
}

\section{Generalized Helices on a Lightlike Cone in 3-Dimensional Lorentz-Minkowski Space \\ ABSTRACT}

In this paper we provide characterizations and give some properties of generalized helices in 3-dimensional LorentzMinkowski space that lie on a lightlike cone. Furthermore, by analyzing their projections, which turn out to be Euclidean or Lorentzian logarithmic spiral, we present their parametrizations. In particular, we also analyze planar generalized helices, that is planar intersections of a lightlike cone.

Key words: Lorentz-Minkowski space, generalized helix, curve of constant slope, lightlike cone

MSC2010: 53A35, 53B30

\section{Introduction}

Generalized helices or curves of constant slope in Euclidean space are curves making a constant angle with a fixed straight line. Hence, they are isogonal trajectories of rulings of a cylindrical surfaces whose rulings are parallel to a fixed direction. A classical Euclidean result states that a curve is a generalized helix if and only if the ratio of its torsion to curvature is constant. Of particular interest it is also to study generalized helices with some additional property, such as lying on certain surfaces, for instance, spheres. In Euclidean space, spherical generalized helices have a property that their orthogonal projections onto a plane normal to their axis appear as epicycloids. In this paper we are interested to analyze the analogous problem of generalized helices in Lorentz-Minkowski 3space with additional property that they lie on a lighlike cone. A lightlike cone $L C(p)$ with a vertex at a point $p$ is, from Euclidean perspective, a rotational cone with vertex $p$
Opće zavojnice na svjetlosnom stošcu u trodimenzionalnom Lorentz-Minkowskijevom prostoru

\section{SAŽETAK}

U radu je dana karaketrizacija općih zavojnica u 3dimenzionalnom Lorentz-Minkowskijevom prostoru koje leže na svjetlosnom stošcu te su predstavljena njihova svojstva. Nadalje, analizirajući njihove projekcije, koje su euklidske ili Lorentzove logaritamske spirale, odredili smo i njihove parametrizacije. Kao specijalan slučaj analizirane su ravninske opće zavojnice, tj. presjeci ravnine i svjetlosnog stošca.

Ključne riječi: Lorentz-Minkowskijev prostor, opća zavojnica, krivulje konstantnog nagiba, svjetlosni stožac

and "vertical" axis. We perform this analysis by analyzing their projection curves.

In Lorentz-Minkowski space, generalized helices are studied in e.g. [4], where their analogous characterization to the Euclidean counterparts are presented. Null helices are studied in [2]. Curves on a lightlike cone are described in terms of their intrinsic curvatures in [3].

\section{Preliminaries}

Let $\mathbb{R}_{1}^{3}$ be a pseudo-Euclidean or Lorentz-Minkowski space, that is, the vector space $\mathbb{R}^{3}$ equipped with the indefinite symmetric bilinear form (a pseudo-scalar product)

$$
\langle x, y\rangle=x_{1} y_{1}+x_{2} y_{2}-x_{3} y_{3} \text {. }
$$

A vector $x$ in the Lorentz-Minkowski 3-space is called spacelike if $\langle x, x\rangle>0$ or $x=0$, timelike if $\langle x, x\rangle<0$ and lightlike if $\langle x, x\rangle=0$ and $x \neq 0$. A timelike vec- 
tor $x=\left(x_{1}, x_{2}, x_{3}\right)$ is said to be positive (resp. negative) if $x_{3}>0\left(x_{3}<0\right)$. The pseudo-norm of a vector $x$ is defined as a real number

$\|x\|=\sqrt{|\langle x, x\rangle|} \geq 0$.

Angle between vectors in $\mathbb{R}_{1}^{3}$ is introduced as follows:

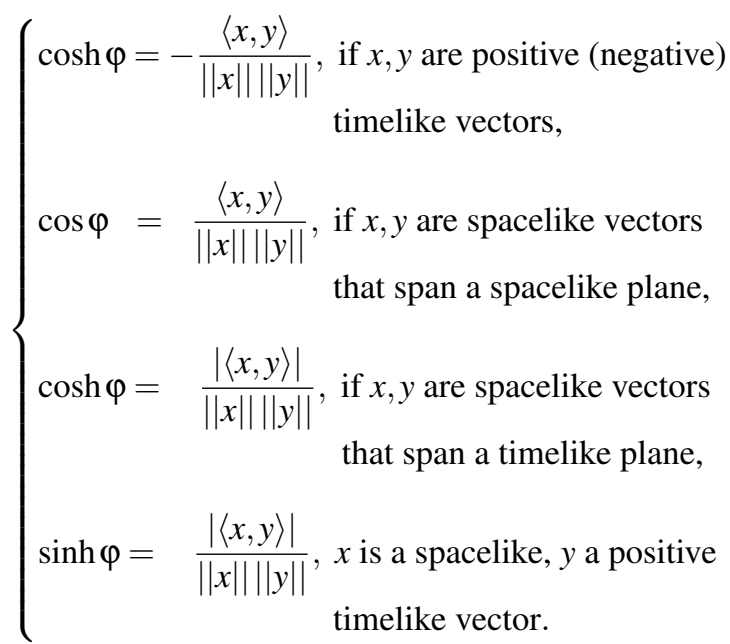

The causal character (spacelike, timelike or lightlike) of a regular curve is determined by the causal character of its velocity vector. The local theory of curves in LorentzMinkowski space can be found in e.g. [4]. For our purposes, it is enough to provide it for spacelike curves only. Let $c: I \subset \mathbb{R} \rightarrow \mathbb{R}_{1}^{3}$ be a spacelike curve parametrized by the arc length. If $c^{\prime \prime}(u)$ is not null, the Frenet frame and the Frenet equations are given by

$$
\left(\begin{array}{l}
T^{\prime} \\
N^{\prime} \\
B^{\prime}
\end{array}\right)=\left(\begin{array}{ccc}
0 & \kappa & 0 \\
-\varepsilon \kappa & 0 & \tau \\
0 & \tau & 0
\end{array}\right)\left(\begin{array}{l}
T \\
N \\
B
\end{array}\right),
$$

where $\varepsilon=\langle N, N\rangle= \pm 1, \kappa(u)=\left\|c^{\prime \prime}(u)\right\|$ is the curvature and $\tau(u)=\varepsilon\left\langle B^{\prime}(u), N(u)\right\rangle$ is the torsion of $c$.

Let $c^{\prime \prime}(u)$ be null for all $u \in I$. These curves are called pseudo-null curves. The principal normal vector is defined as $N(u)=c^{\prime \prime}(u)$. The binormal vector $B(u)$ is the null vector orthogonal to $T$ satisfying $\langle N, B\rangle=1$. Then the Frenet equations are

$$
\left(\begin{array}{c}
T^{\prime} \\
N^{\prime} \\
B^{\prime}
\end{array}\right)=\left(\begin{array}{ccc}
0 & \kappa & 0 \\
0 & \tau & 0 \\
-\kappa & 0 & -\tau
\end{array}\right)\left(\begin{array}{c}
T \\
N \\
B
\end{array}\right) .
$$

Here $\tau=\left\langle N^{\prime}, B\right\rangle$ is the so-called pseudo-torsion, [4, 5]. The curvature $\kappa$ can be either 0 (a curve is a straight line), or $\kappa=1$.

\section{Curves lying on a lightlike cone}

A lightlike cone $L C(p)$ with the vertex $p$ in LorentzMinkowski space $\mathbb{R}_{1}^{3}$ is a quadratic surfaces defined as

$L C(p)=\left\{q \in \mathbb{R}_{1}^{3}:\langle q-p, q-p\rangle=0\right\}$.

It is a (Euclidean) rotational cone with the axis $x_{3}$, and a ruled surface with lightlike generators (rulings). As a surface in $\mathbb{R}_{1}^{3}$ it inherits a degenerated metric of rank 1 (an isotropic metric), therefore it is a lightlike surface. The lightlike cone with the vertex at 0 is simply denoted by $L C$. If a curve $c$ lies on a lightlike cone $L C(p)$, then $\langle c(s)-p, c(s)-p\rangle=0$ and therefore $c(s)-p$ is a lightlike (null) vector. This implies $\left\langle c(s)-p, c^{\prime}(s)\right\rangle=0$, that is, $c^{\prime}(s)$ is orthogonal to a lightlike vector $c(s)-p$. Then $c^{\prime}(s)$ is either lightlike or spacelike (excluding zero vector due to regularity of a curve). In the case when $c^{\prime}(s)$ is lightlike, it needs to be collinear to $c(s)-p$, which would imply that $c$ is a null straight line (the only lightlike vectors orthogonal to a lightlike vector are collinear vectors). In the case when $c^{\prime}(s)$ is a spacelike vector, we can assume that it is parametrized by arc length, $\left\langle c^{\prime}, c^{\prime}\right\rangle=1$. The vector $c^{\prime \prime}(s)$ can be either spacelike, timelike or lightlike. Curves with the last property are pseudo-null curves.

For curves lying on $L C(p)$, the following result holds.

Theorem 1 A curve $c$ lies on a cone $L C(p)$ centered at $p$ if and only if

1. $c$ is a spacelike curve with $c^{\prime \prime}$ spacelike or timelike that satisfies (when parametrized by arc length)

$\rho \tau= \pm \rho^{\prime}$

where $\rho=\frac{1}{\kappa}$;

2. $c$ is a spacelike curve with $c^{\prime \prime}$ lightlike if and only if $c$ is a Lorentzian circle in a lightlike plane (that is, a Euclidean parabola with the axis parallel to the lightlike direction);

3. $c$ is a lightlike curve if and only if $c$ is a generator line of a ruled surface $L C(p)$.

Proof. To prove the first statement, we recall that $\langle c(s)-p, c(s)-p\rangle=0$ implies $\langle c(s)-p, T(s)\rangle=0$. Differentiating the previous equation and using the Frenet formulas (3) we get

$\langle c(s)-p, N(s)\rangle=-\rho(s),\langle c(s)-p, B(s)\rangle=\varepsilon \frac{\rho^{\prime}}{\tau}$.

Therefore, if we write $c(s)-p=\alpha T(s)+\beta N(s)+\gamma B(s)$, we can conclude

$c(s)-p=-\rho N(s)-\varepsilon \frac{\rho^{\prime}}{\tau} B$. 
Furthermore, since $c(s)-p$ is a null vector, we have

$$
\rho^{2}=\left(\frac{\rho^{\prime}}{\tau}\right)^{2}
$$

which was to prove. For the second statement we recall that a pseudo-null curve is planar and lies in a lightlike plane, [1]. A lightlike plane contains only one null direction, that is, a direction parallel to one null ruling of $L C(p)$. Therefore, a curve $c$ is planar section of $L C(p)$ yielding as an intersection a Euclidean parabola with the axis parallel to the null direction, that is, a Lorentzian circle in a lightlike plane. Finally, the last statement is obvious.

Remark 1 Notice that among the curves described by the case (1) in the previous theorem, there are also other planar sections beside Euclidean parabola described in (2), that is curves that satisfy $\tau=0 . \quad B y$ the condition (5) their curvature is $\kappa=$ const. $\neq 0$. Therefore, they are Euclidean (resp. Lorentzian) circles in spacelike (resp. timelike) planes, that is, from Euclidean perspective, ellipses and equilateral hyperbolas (see Figure 1).


Figure 1: Lorentzian circle in spacelike, timelike and lightlike plane, respectively.

\section{Generalized helices and their plane pro- jections}

A generalized helix is a regular unit speed curve (parametrized by the arc length or a pseudo-arc length) for which there exists a constant vector $u \in \mathbb{R}_{1}^{3}, u \neq 0$, such that

$\langle T, u\rangle=$ const. $=: \alpha$.

If a spacelike or a timelike curve $c$ is a generalized helix then

$$
\frac{\tau}{\kappa}=\text { const. }=: \text { A, }
$$

and conversely, for a curve $c$ with non-lightlike normal vectors, [4]. A direction determined by $u$ is called the axis of a helix.
We allow also curves satisfying $A=0$, that is, planar curves, to belong to the class of generalized helices. The constant vector $u$ from the definition is the unit normal of the plane in which the curves lie, therefore $\alpha=0$.

In this section we are interested in relation of a generalized helix and its projection curve onto a certain plane in Lorentz-Minkowski space. For this purpose we consider general non-lightlike planes as projection planes. Let a plane $\pi$ be determined by its unit normal vector $u$, which is spacelike (resp. timelike) when a plane $\pi$ is timelike (resp. spacelike). We denote $\delta=\langle u, u\rangle= \pm 1$. Then a projection curve $\tilde{c}$ of $c$ onto a plane $\pi$ is given by

$\tilde{c}=c-\delta\langle c, u\rangle u$.

If the initial curve $c$ is spacelike, the following result holds:

Theorem 2 Let c be a (unit speed) spacelike generalized helix with respect to a unit spacelike or timelike vector $u$. Let $\tilde{c}$ be the projection of $c$ onto a plane orthogonal to $u$. Then $\tilde{c}$ has a constant speed. Furthermore, if the principal normals of $c$ and $\tilde{c}$ are of the same causal non-null character, the curvature of $c$ and of $\tilde{c}$ are related by

$\tilde{\kappa}^{2}\left(1-\delta \alpha^{2}\right)^{2}=\kappa^{2}$.

Proof. Let $s$ be the arc length parameter of $c$ and $\tilde{s}$ of $\tilde{c}$. Differentiating (8) with respect to $s$ yields

$T=\tilde{T} \frac{d \tilde{s}}{d s}+\delta\langle T, u\rangle u$

Therefore, since $\tilde{T}$ and $u$ are orthogonal,

$\langle T, T\rangle=\langle\tilde{T}, \tilde{T}\rangle\left(\frac{d \tilde{s}}{d s}\right)^{2}+\delta \alpha^{2}$.

Moreover, we have and $\langle T, T\rangle=1$, and $\langle\tilde{T}, \tilde{T}\rangle= \pm 1$, since the causal character of $\tilde{c}$ is not known. Therefore we have

$\pm\left(\frac{d \tilde{s}}{d s}\right)^{2}=1-\delta \alpha^{2}$.

Moreover, the speed of $\tilde{c}$ is given by

$\left\|\frac{d \tilde{c}}{d s}\right\|=\left\|\tilde{T} \frac{d \tilde{s}}{d s}\right\|=\sqrt{\left|1-\delta \alpha^{2}\right|}=$ const.

which implies that $\tilde{c}$ is of constant speed (with respect to $s)$.

To prove equation (9), we proceed as follows. First we notice that by (6) from the definition of a generalized helix we have

$$
\langle N, u\rangle=0 .
$$

Now, by differentiating (10) and using the previous equation, we get

$\kappa(s) N(s)=\tilde{\kappa}(\tilde{s}) \tilde{N}(\tilde{s})\left(\frac{d \tilde{s}}{d s}\right)^{2}$, 
where $\tilde{\kappa}$ is the curvature of $\tilde{c}$ (as a curve in Euclidean or Lorenzian plane, $\mathbb{R}^{2}$ or $\mathbb{R}_{1}^{2}$ ). We can notice that the previous equation implies that $N$ and $\tilde{N}$ should be collinear, and therefore of the same causal character. In that case, by taking the pseudo-scalar square of the previous equation we get

$\kappa(s)^{2}=\tilde{\kappa}(\tilde{s})^{2}\left(\frac{d \tilde{s}}{d s}\right)^{4}$.

Now (11) implies that the equation (9) holds. Note that otherwise, when $N$ and $\tilde{N}$ are not of the same causal character, it would not be possible for the relation (9) to be satisfied in real numbers.

Remark 2 The relation (9) between the curvatures of an initial curve and its projection is valid only under the assumption that their principal normals are of the same causal character. For example, the relation holds if a curve $c$ with $\kappa=$ const $. \neq 0, \tau=0$, that is a circle in $\mathbb{R}_{1}^{3}$, is projected onto the plane of the same character as the plane in which it lies: if $c$ is a circle in a spacelike plane (an ellipse from the Euclidean perspective), then its projection onto xy-plane is a "real" circle in Euclidean plane, having constant curvature $\tilde{\kappa}$.

In the next result we discuss the causal character of the projection of a spacelike generalized helix.

Theorem 3 Let $c$ be a (unit speed) spacelike generalized helix with respect to a unit spacelike or timelike vector $u$. Then $\tilde{c}$ is:

1. spacelike, if u timelike or if $u$ is spacelike such that $u$ and $T$ span a spacelike plane;

2. timelike, if $u$ is spacelike such that $u$ and $T$ span a timelike plane.

Proof. Statements follow from the analysis of the speed of a projection, $\left\langle\tilde{c}^{\prime}, \tilde{c}^{\prime}\right\rangle=1-\delta \alpha^{2}$, where derivative is taken with respect to $s$. If $u$ is a timelike unit vector, then $\delta=-1$, which implies $\left\langle\tilde{c}^{\prime}, \tilde{c}^{\prime}\right\rangle=1+\alpha^{2}$. Hence the projection $\tilde{c}$ is spacelike (which is actually obvious as $\tilde{c}$ is a projection onto a spacelike plane). To prove other statements, we relate the constant $\alpha$ in the definition (6) to the angle between the initial curve $c$ and $u$, see (2). If $u, T$ are spacelike vectors that span a spacelike plane, then we can interprete $\alpha$ as $\alpha=\cos \varphi$, for some real number $\varphi$; if they span a timelike plane, then $|\alpha|=\cosh \varphi$. Therefore, in the first case of the Theorem we have $\left\langle\tilde{c}^{\prime}, \tilde{c}^{\prime}\right\rangle=1-\alpha^{2}=1-\cos ^{2} \varphi=\sin ^{2} \varphi>0$, and in the second case $\left\langle\tilde{c}^{\prime}, \tilde{c}^{\prime}\right\rangle=1-\alpha^{2}=1-\cosh ^{2} \varphi=-\sinh ^{2} \varphi<0$. By these relations the causal character of $\tilde{c}$ is determined.
Remark 3 Let us discuss the case when a curve $c$ is lightlike. The lightlike (null) generalized helices are those having a pseudo-torsion (lightlike curvature) constant [2, 4]. There are three types of null helices parametrized by pseudo-arc parameter, those congruent to $c(s)=\left(\frac{1}{a^{2}} \cos (a s), \frac{1}{a^{2}} \sin (a s), \frac{s}{a}\right), \quad c(s)=$ $\left(-\frac{s}{a}, \frac{1}{a^{2}} \cosh (a s), \frac{1}{a^{2}} \sinh (a s)\right)$ or to so-called null cubics $c(s)=\left(\frac{s^{3}}{4}-\frac{s}{3}, \frac{s^{2}}{2}, \frac{s^{3}}{4}+\frac{s}{3}\right)$. Their axes are timelike, spacelike and lightlike, respectively. None of them are curves lying on a lightlike cone in Lorentz-Minkowski space.

\section{Generalized helices lying on a lightlike cone $L C(p)$}

Theorem 4 Let $c$ be a (unit-speed) generalized helix with respect to a unit vector $u$ that lies on a lightlike cone $L C(p)$. Then the curvature and the torsion of $c$ are given by

$\kappa(s)= \pm \frac{1}{A s}, \tau(s)= \pm \frac{1}{s}$.

The curvature $\tilde{\mathrm{\kappa}}(\tilde{s})$ of a projection $\tilde{c}$ of $c$ on the plane orthogonal to $u$ is given by

$\tilde{\kappa}(\tilde{s})=\frac{1}{a \tilde{s}}$, i.e. $\tilde{\rho}(\tilde{s})=a \tilde{s}$,

where $a^{2}=A^{2}\left|1-\delta \alpha^{2}\right|$.

In particular, if $A=0$, that is, if $c$ is planar, then $\kappa(s)=$ const. $\neq 0, \tau=0$, and therefore $\tilde{\kappa}(\tilde{s})=$ const.$\neq 0$.

Proof. If $c$ is a unit-speed on $L C(p)$, then $c$ is a spacelike curve whose curvature and torsion satisfy (5). Then $\rho^{\prime 2}=A^{2}$, that is, $\rho= \pm A s+c$ and the assertion follows (by neglecting without a loss of generality a constant $c$ ). The curvature of a projection $\tilde{c}$ is obtained from (9). Furthermore, in particular if $A=0$, then $\alpha=0$, (9) implies $\kappa(s)=\tilde{\kappa}(\tilde{s})=$ const .

Remark 4 Pseudo-null curves lying on a lightlike cone, that is Euclidean parabolas in lightlike planes (with pseudo-torsion $\tau=0$ ), are also curves of constant slope by definition, but we do not consider their projection since they have null principal normals.

Our next goal is to analyze plane projections of generalized helices lying on a lightlike cone in Lorentz-Minkowski 3space. Because of a special position of a lightlike cone $L C(p)$ (having $x_{3}$ as its axis, usually depicted as vertical), we are interested in projections onto a spacelike $x y$-plane and Lorentzian $x z$-plane. Let $c$ be a curve on a lightlike cone (spacelike curve parametrized by arc length) and $u$ a timelike vector $(\delta=-1)$. The constant $\alpha$ can be interpreted in terms of an angle as $\alpha=\langle T, u\rangle=\sinh \varphi$. Then we have $\left\langle\tilde{c}^{\prime}, \tilde{c}^{\prime}\right\rangle=1-\delta \alpha^{2}=\cosh ^{2} \varphi$. The projection $\tilde{c}$ onto a spacelike plane orthogonal to $u$ is a spacelike curve with 
the arc length parameter $\tilde{s}=(\cosh \varphi) s$. The curvature $\tilde{\kappa}$ is given by (14) as

$\tilde{\kappa}(\tilde{s})= \pm \frac{1}{(A \cosh \varphi) \tilde{s}}=\frac{1}{a \tilde{s}}$,

where $a= \pm A \cosh \varphi$.

We can reconstruct the projection curve $\tilde{c}$ from its natural equation (14). In the case when the projection plane is $x y$-plane, we introduce

$t(\tilde{s})=\int_{\tilde{s}_{0}}^{\tilde{s}} \tilde{\mathrm{K}}(\tilde{s}) d \tilde{s}=\int_{\tilde{s}_{0}}^{\tilde{s}} \frac{1}{a \tilde{s}} d \tilde{s}=\frac{1}{a} \ln |b \tilde{s}|$,

where $b$ is a real constant. The curve $\tilde{c}$ can be written as

$\tilde{c}(\tilde{s})=\left(\int_{\tilde{s}_{0}}^{\tilde{s}} \cos t(\tilde{s}) d \tilde{s}, \int_{\tilde{s}_{0}}^{\tilde{s}} \sin t(\tilde{s}) d \tilde{s}\right)$.

By calculating these integrals and using (15) we get the following parametrization of the curve $\tilde{c}$

$x(t)=\frac{a}{b\left(1+a^{2}\right)} e^{a t}(a \cos t+\sin t)$,
$y(t)=\frac{a}{b\left(1+a^{2}\right)} e^{a t}(-\cos t+a \sin t)$.

We can notice that this curve is a logarithmic spiral (see Figure 2, right). Now, having a projection, the remaining coordinate of a curve $c$ lying on a lightlike cone $L C$ is calculated as

$z(t)=\frac{a}{b \sqrt{1+a^{2}}} e^{a t}$.

See Figure 2.
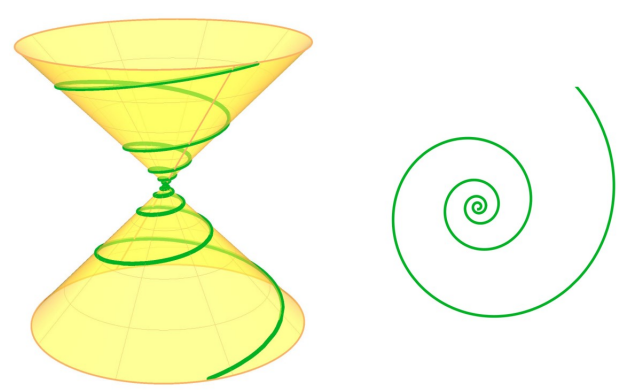

Figure 2: A generalized helix on a cone and its projection on xy-plane

Now, consider $u$ to be a spacelike vector $(\delta=1)$, in particular $u=(0,1,0)$. The projection plane is a timelike $x z-$ plane.

If $c^{\prime}$ and $u$ span a spacelike plane, a projection curve $\tilde{c}$ is spacelike. Then $\alpha$ can be interpreted as $\cos \varphi, \varphi \in \mathbb{R}$ (see (2)). Then we have $\left\langle\tilde{c}^{\prime}, \tilde{c}^{\prime}\right\rangle=\sin ^{2} \varphi$, and for the arc length parameters the following holds $\tilde{s}=\sin \varphi s$. The curvature $\tilde{\kappa}$ satisfies (14) with $a=A \sin \varphi$. The spacelike curve $\tilde{c}$ in a timelike plane can be reconstructed from (see [4])

$\tilde{c}(\tilde{s})=\left(\int_{\tilde{s}_{0}}^{\tilde{s}} \cosh t(\tilde{s}) d \tilde{s}, \int_{\tilde{s}_{0}}^{\tilde{s}} \sinh t(\tilde{s}) d \tilde{s}\right)$,

where $t(\tilde{s})$ is given by (15). By calculating these integrals and using (15) we get the following parametrization of the curve $\tilde{c}$ with $a \neq 1$

$x(t)=\frac{a}{b\left(a^{2}-1\right)} e^{a t}(-\sinh t+a \cosh t)$,

$z(t)=\frac{a}{b\left(a^{2}-1\right)} e^{a t}(a \sinh t-\cosh t)$.

It would be a Lorentzian logarithmic spiral (see Figure 3, right). Furthermore, for the initial curve we need the coordinate

$y(t)=\frac{a}{b \sqrt{1-a^{2}}} e^{a t}$.

See Figure 3.

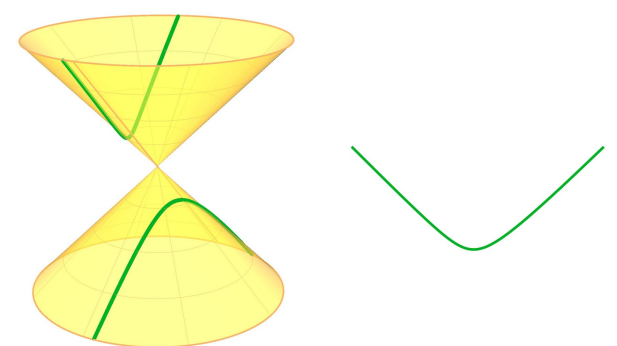

Figure 3: A generalized helix on a cone and its projection on xz-plane

In the case when $a=1, b=1$ we have

$x(t)=\frac{1}{2}\left(\frac{e^{2 t}}{2}+t\right)$,

$z(t)=\frac{1}{2}\left(\frac{e^{2 t}}{2}-t\right)$

The coordinate $y$ is given by

$y^{2}(t)=-\frac{1}{2} e^{2 t} t$

which restricts to $t<0$. See Figure 4 .
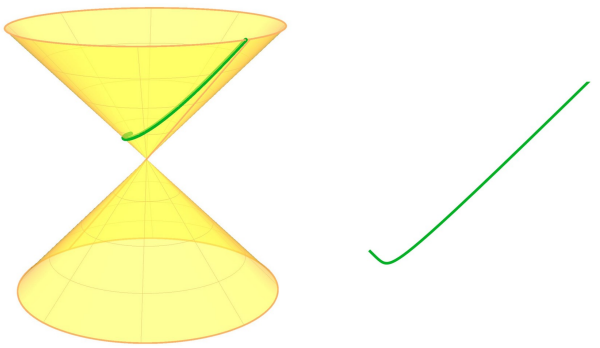

Figure 4: A generalized helix on a cone and its projection on $x z$-plane 
Finally, if $u$ is spacelike, and $c^{\prime}$ and $u$ span a timelike plane, then by (2) we have $|\alpha|=\cosh \varphi, \varphi \in \mathbb{R}$. Then we have $\left\langle\tilde{c}^{\prime}, \tilde{c}^{\prime}\right\rangle=-\sinh ^{2} \varphi$, and for the arc length parameters $\tilde{s}=(\sinh \varphi) s$. The curvature $\tilde{\kappa}$ satisfies (14) with $a= \pm A \sinh \varphi$. The timelike curve $\tilde{c}$ in a timelike plane can be reconstructed from

$\tilde{c}(\tilde{s})=\left(\int_{\tilde{s}_{0}}^{\tilde{s}} \sinh t(\tilde{s}) d \tilde{s}, \int_{\tilde{s}_{0}}^{\tilde{s}} \cosh t(\tilde{s}) d \tilde{s}\right)$

where $t(\tilde{s})$ is given by (15). Lorentzian logarithmic spirals obtained in this case are the same as in the previous, but with the $x$ and $z$ coordinates interchanged.

\section{References}

[1] S. Chino, S. IzumiYA, Lightlike developables in Minkowski 3-space, Demonstr. Math., 43(2) (2010), 387-399.

[2] J. Inoguchi, S. LeE, Null curves in Minkowski 3space, Int. Electron. J. Geom. 1 (2008) 40-83.

[3] H. LIU, Curves in the Lightlike Cone, Beitr. Algebra Geom. [Contributions to Algebra and Geometry] 45 (2004), 291-303.

[4] R. López, Differential Geometry of Curves and Surfaces in Lorentz-Minkowski space, Int. Electron. J. Geom. 7 (2014), 44-107.

[5] J. Walrave, Curves and Surfaces in Minkowski Space, Ph. D. Thesis, K. U. Leuven, Faculteit Der Wetenschappen, 1995.
Finally, as we have already commented, when projections curves are circles in $x y$ - or $x z$-planes, that is, curves having a constant curvature $\tilde{\kappa}$, then by (7), their torsion $\tau$ is also constant, meaning they are helices lying on a circular cylinder as well. Then they should be planar curves, that is planar intersections of a lighlike cone (Euclidean circles or hyperbolas).

We can also note, that contrary to Euclidean case, where the projections of cone helices are logarithmic spirals and circles only, in Lorentz-Minkowski case we have other classes of cone helices as well, those whose projections are Lorentzian logarithmic spirals and hyperbolas, and those lying in lightlike planes (parabolas).

\section{Željka Milin Šipuš}

orcid.org/0000-0002-0726-3335

e-mail: zeljka.milin-sipus@math.hr

Faculty of Science, University of Zagreb

Bijenička cesta 30, HR-10000 Zagreb, Croatia

\section{Ivana Protrka}

orcid.org/0000-0001-6616-3206

e-mail: ivana.protrka@rgn.hr

Faculty of Mining, Geology and Petroleum

Engineering, University of Zagreb

Pierottijeva 6, HR-10000 Zagreb, Croatia

\section{Ljiljana Primorac Gajčić}

orcid.org/0000-0002-8460-3196

e-mail: Iprimora@mathos.hr

Department of Mathematics, University of Osijek

Trg Ljudevita Gaja 6, HR-31000 Osijek, Croatia 